\title{
Fabrication and Characterization of $S$-Benzyldithiocarbazate Schiff Base Microcrystals by a Reprecipitation Method for Enhanced Antibacterial Activity
}

\author{
Nur Halilatul Sadiqin Omar Ali ${ }^{1}$, Siti Norliza Sa'adah Abdul Rahman ${ }^{1}$, Hajar Azirah Adol ${ }^{1}$, \\ Malai Haniti Sheikh Abdul Hamid ${ }^{1}$, Hussein Taha ${ }^{2}$, Mohd Yameany Haji Rosli ${ }^{3}$, Narayana \\ Thotagamuge Roshan Nilantha Kumara ${ }^{4}$, Abdul Hanif Mahadi ${ }^{4}$, Anwar Usman ${ }^{1}$ \\ ${ }^{1}$ Chemical Sciences, Faculty of Science, Universiti Brunei Darussalam, Jalan Tungku Link, Gadong BE1410, \\ Brunei Darussalam \\ ${ }^{2}$ Environmental and Life Sciences, Faculty of Science, Universiti Brunei Darussalam, Jalan Tungku Link, Gadong \\ BE1410, Brunei Darussalam \\ ${ }^{3}$ Geosciences, Faculty of Science, Universiti Brunei Darussalam, Jalan Tungku Link, Gadong BE1410, Brunei \\ Darussalam \\ ${ }^{4}$ Centre for Advanced Material and Energy Sciences, Universiti Brunei Darussalam, Jalan Tungku Link, \\ Gadong BE1410, Brunei Darussalam
}

\begin{abstract}
In this study, the microcrystallization of the 2-thiophenecarboxaldehyde Schiff base of $S$ benzyldithiocarbazate (i.e. benzyl (2E)-2-[(thiophen-2-yl)methylidene]hydrazine-1-carbodithioate [TASBnDTC]) was fabricated by a reprecipitation method in an organic solvent-water system using different crystallization parameters, including temperature and the concentration of the target compound. The size, anisotropy, crystalline phase, and surface morphology of the TASBnDTC microcrystals were characterized by dynamic light scattering and scanning electron microscopy. The stability of the Schiff base microcrystals was also evaluated. Different sizes of surfactantdispersed TASBnDTC microcrystals $(1505,2194$, and $2447 \mathrm{~nm})$ were fabricated from three different concentrations of the Schiff base $(0.001 \mathrm{M}, 0.002 \mathrm{M}$, and $0.003 \mathrm{M}$, respectively) in an acetone-water system. The TASBnDTC microcrystals were also evaluated by X-ray powder diffraction and were found to differ slightly in molecular form but were otherwise similar, irrespective of the different TASBnDTC concentrations. The synthesized Schiff bases and their microcrystals were also screened for their antibacterial activities against four different grampositive and gram-negative bacterial strains (Escherichia coli, Bacillus subtilis, Pseudomonas aeruginosa, and Staphylococcus aureus) using the agar well diffusion method. The growth inhibition was enhanced by 8.0 to $10.75 \mathrm{~mm}$ against the four bacteria by TASBnDTC microcrystals compared to the bulk molecular form, which showed no inhibitory activity at all. However, the inhibition was less that that achieved with the standard streptomycin antibiotic, which gave zones of inhibition of 18.0 to $23.0 \mathrm{~mm}$ against the four bacterial strains. Overall, the Schiff base microcrystals show potential for use in various biological applications. They also have potential physical and optical applications due to their high surface-to-volume ratio and the molecular alignment on the surface of the microcrystals.
\end{abstract}

Keywords: Antibacterial activity; Nanoscience; Organic microcrystal; Reprecipitation method; Schiff base 


\section{Introduction}

In recent times, nanotechnology has been integrated in many parts of our everyday lives. The fabrication of organic and inorganic nano- and microcrystals is one area of growing interest from the perspectives of both fundamental science and applications (Poerwadi et al., 2020; Strokova et al., 2020). However, organic microcrystals have received little attention so far because of difficulties with their characterization and the thermal instability of most of the organic compounds.

Organic microcrystals with sizes of several tens of nanometers to the sub-micrometer crystal size have been fabricated by either "top-down" approaches, such as milling methods (Li et al., 2020), or by "bottom-up" approaches, such as reprecipitation (Kasai et al., 1992; 2012) and supercritical fluid crystallization (SCFC) (Komai et al., 1999). In the "top-down" approaches, the microcrystals are fabricated by size reduction techniques, whereas in the "bottom-up" approaches, the microcrystals are fabricated from atoms or molecules by synthetic chemistry or self-assembly processes. The most common "bottom-up" approach is the so-called reprecipitation method first reported by Kasai et al. (1992). The reprecipitation method has many advantages, as it is simple, quick, and inexpensive. In addition, it is applicable to many kinds of organic compounds. However, this method cannot be applied in the case of water-soluble organic dyes or barely water-soluble organic compounds, such as pigments. However, Tripathy et al. (1998) recently introduced a new approach for fabricating well-defined organic microcrystals through electrostatic selfassembly. Similarly, Baba et al. (2000) introduced a novel microwave-irradiation process to improve the conventional reprecipitation method.

Most of the current research focuses on the preparation of free-standing organic microcrystals, and far less work has been conducted on inorganic semiconductor microcrystals. These are organic microcrystals with sizes ranging from a few tens to a few hundred nanometers, and they have received substantial attention in the past few decades (Tripathy et al., 1998; Oikawa et al., 2000). This interest arises largely from the observation that their unique size-dependent optical, electronic, and chemical properties differ from those of either their single molecules or their bulk crystals (Jortner and Rao, 2002; Chiang et al., 2012; Indrasti et al., 2020). The excitement of nanoscience and the potential applications of these microcrystals are now driving intensive research activities, and organic nano-/microcrystals are now finding a broad range of applications in the areas of lithography (Magdassi and Moshe, 2003), organic photoconductors (Miyashita et al., 2008) and pharmaceutical formulations for drug delivery and biomedical applications (Kumar and Lal, 2014).

Typically, organic microcrystals have a high surface energy and large curvature, which changes the chemical nature of the organic molecules on the surface and renders the small organic microcrystals thermodynamically unstable. For this reason, obtaining organic nano-/microcrystals even with sizes below 100 nanometers is very difficult, and this size is still much larger than that of semiconductor or noble metal nano-/microcrystals (Li et al., 2017). Therefore, much research has focused on the use of additives, capping agents, or inorganic or polymer matrices to control the size and shape of organic microcrystals (Abyan et al., 2005). One approach has been the use of Schiff bases containing imine group $(-\mathrm{CH}=\mathrm{N}-)$ as these bases have structural peculiarities and wide pharmacological and biological applications (Fasina et al., 2013; Mondal et al., 2017).

Schiff bases are usually formed by the condensation of a primary amine with a carbonyl compound (an aldehyde or ketone) in the presence of an acid catalyst. A careful survey of the literature shows only limited reports on the fabrication of Schiff-base micro-scale crystals by reprecipitation methods (Mahajan et al., 2017; Patil et al., 2017; Kumar et al., 
2019). In recent years, interest has grown in the chemistry of $S$-alkyl/aryl dithiocarbazates owing to their potential biological activities and their great behavior as intermediates in organic reactions (Malik et al., 2020). To the best of our knowledge, the fabrication of this type of Schiff base by a reprecipitation method has not been reported previously. Therefore, this paper summarizes the details of a preliminary investigation on the fabrication, characterization and antibacterial studies of a $S$-benzyldithiocarbazate Schiff base and its microcrystals.

\section{Methods}

\subsection{Materials and Reagents}

2-Thiophenecarboxaldehyde, Mueller-Hinton agar, nutrient broth, and streptomycin sulfate were purchased from Sigma-Aldrich Co. (Sigma Aldrich Chemie GmbH, München, Germany). Acetone ACS reagent (dielectric constant $(\varepsilon)=20.7$, bp. $56^{\circ} \mathrm{C}$ ) and cetrimonium bromide (CTAB) were purchased from Merck (Merck Millipore, Darmstadt, Germany). All chemicals were used without any further purification. Ultrapure water was used throughout the experiments. $S$-benzyldithiocarbazate used was prepared following a previously reported procedure (Raj et al., 2000).

\subsection{Characterization}

All surfactants were mixed independently in ultrapure water and each mixture was sonicated using a Zoex Elmasonic E device (E $180 \mathrm{H})$ at a frequency of $37 \mathrm{kHz}$. The electronic spectra of the Schiff base and its microcrystals were recorded in acetone on a Shimadzu (UV-1601) UV-Vis spectrophotometer. The size distribution and surface zeta potential were evaluated by dynamic light scattering (DLS) measurements using a Brookhaven DLS digital correlator (BI 9000) equipped with a diode-pumped laser (532 nm; $10 \mathrm{~mW})$. The surface morphology of the Schiff base and its microcrystals were inspected by scanning electron microscopy (SEM) (JEOL JSM67005). For this measurement, the dispersed microcrystals in water were collected and pasted onto carbon paste for SEM imaging with at least 105× magnification. X-ray powder diffraction (XRD) patterns of the prepared microcrystals were recorded on a Shimadzu diffractometer (XRD-700) with $\mathrm{Cu}$ K $\alpha$ radiation $(\lambda=1.54 \AA$ ).

\subsection{Preparation of the Schiff Base and its Microcrystals}

This study focused on the Schiff base formed from the condensation of 2thiophenecarboxaldehyde with $S$-benzyldithiocarbazate. Benzyl (2E)-2-[(thiophen-2yl)methylidene]hydrazine-1-carbodithioate (TASBnDTC), as shown in Scheme 1, was prepared as previously reported by our group (Ali et al., 2020).<smiles>O=Cc1cccs1</smiles>

2-thiophenecarboxaldehyde

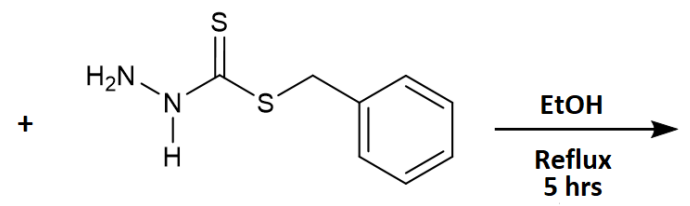

S-benzyldithiocarbazate<smiles>S=C(N/N=C/c1cccs1)SCc1ccccc1</smiles>

Yield 57\% TASBnDTC

Scheme 1 Synthetic route for the synthesis of TASBnDTC

Microcrystals of TASBnDTC were fabricated by the reprecipitation method (Figure 1) following the preparation for the synthesis of organic microcrystals reported by Kasai et al. (1992; 2012), with some modifications. The Schiff base was dissolved in acetone, which is a good solvent, and then $500 \mu \mathrm{L}$ of the Schiff base solution was quickly injected, using a microsyringe, into $10 \mathrm{~mL}$ of a $0.05 \% \mathrm{CTAB}$-ultrapure water mixture, which is a poor solvent, 
with vigorous stirring at room temperature. The concentration of the Schiff base compound in the solution ranged between 0.0005 and $0.003 \mathrm{M}$. The solution immediately turned cloudy and yellow in color, indicating the formation and dispersion of pale yellow TASBnDTC microcrystals. The resulting dispersion was continuously and vigorously stirred (800 rpm) for 10 minutes, and the stable dispersion was then subjected to various characterization methods. All experiments were carried out at room temperature, unless otherwise stated.

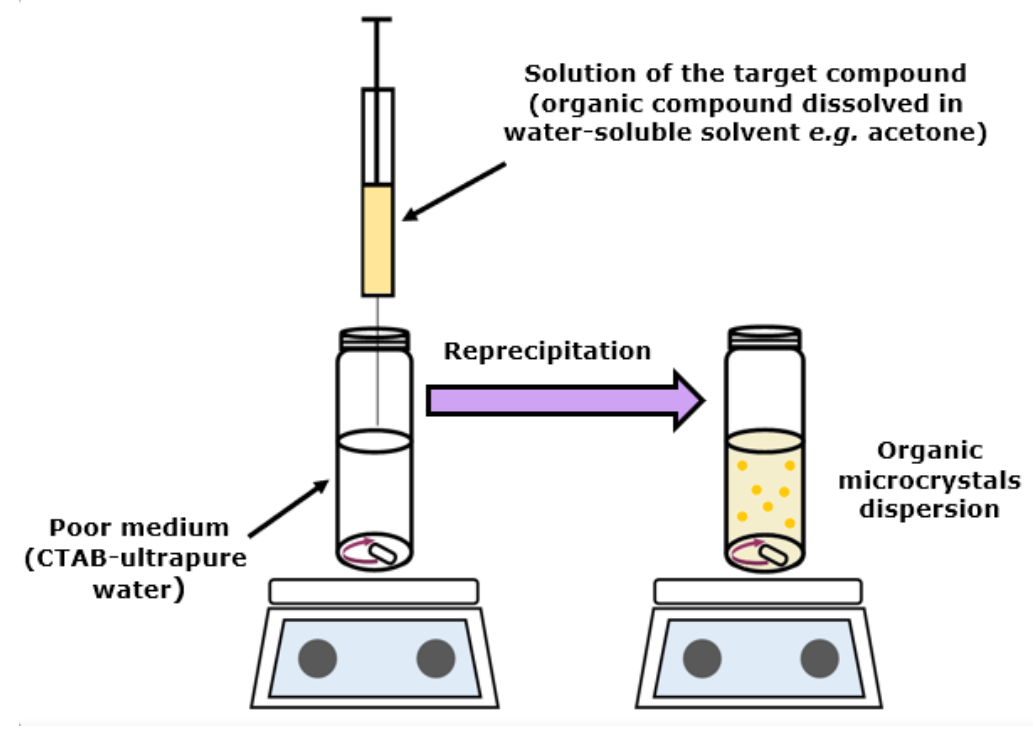

Figure 1 Schematic representation of the experimental setup, modified from the reprecipitation method reported by Kasai et al. (2012) (equipment/instrument used: Hamilton microsyringe; Thermolyne ceramic 2 hot plate stirrer; BRAND cylindrical magnetic stirrer bar, $20 \times 6 \mathrm{~mm}$; and screw top clear glass vial $15 \mathrm{~mL}$ )

\subsection{Evaluation of Antibacterial Activity}

The antibacterial activity of the synthesized Schiff base and its microcrystals were determined using the agar well diffusion method reported by Jahangirian et al. (2013), with a slight modification. Briefly, $100 \mu \mathrm{L}$ of pathogenic bacteria were grown in $7 \mathrm{~mL}$ fresh nutrient broth by incubating and agitating them at $150 \mathrm{rpm}$ overnight in a water bath at the optimal growth temperature of $37^{\circ} \mathrm{C}$. A $200 \mu \mathrm{L}$ volume of the microbial suspension (first adjusted to a 0.5 McFarland turbidity standard) was spread onto Mueller-Hinton agar plates. A $40 \mu \mathrm{L}$ volume of the solution to be tested $(2 \mathrm{mg} / \mathrm{mL})$ for antibacterial activities was gently pipetted into the wells. Dimethyl sulfoxide (DMSO) and ultrapure water were used as negative standards, while streptomycin sulfate was used as a positive standard. Plates with gram-positive bacteria (Staphylococcus aureus ATCC 29213 and Bacillus subtilis ATCC 1174) and gram-negative bacteria (Escherichia coli ATCC 11775 and Pseudomonas aeruginosa ATCC 27853 ) were incubated at $37^{\circ} \mathrm{C}$ for $24 \mathrm{~h}$. After the incubation, the diameter of the inhibition zone was measured.

\section{Results and Discussion}

\subsection{Dynamic Light Scattering (DLS)}

Microcrystals of TASBnDTC are very prone to aggregation. As a result, without the use of any surfactant, the microcrystals formed were all in the range of ten thousand nanometers in diameter. The surfactant was mixed in the ultrapure water used in 
reprecipitation method. Henceforth, in all parameters being studied, $0.05 \%$ wt of CTAB in ultrapure water was used as the poor solubility medium.

The DLS data shown in Figure 2a indicated that a broad range of Schiff base crystal sizes can be attained by varying the concentration of the target compound dissolved in acetone. Six different concentrations of the Schiff base were studied $(0.0005,0.0010,0.0015,0.0020$, 0.0025 , and $0.0030 \mathrm{M}$ ). A distinct trend is evident, where the microcrystal size increases gradually with the concentration of the target compound dissolved in acetone. This strong dependence of microcrystal size on the concentration of the Schiff base indicates that the nature of the interaction between the organic solvent and water plays a vital role in determining the microcrystal size. This suggests the possibility that the mechanism governing the microcrystallization process involves both the rate and the efficiency of the dispersion of the organic solvent in water, as well as the intermolecular interactions of the Schiff base molecules (Rahman, 2018). Hence, once the organic solvent is rapidly injected into the ultrapure water, micrometer-sized droplets are dispersed in the water, and a liquid-liquid phase transition process subsequently occurs. For the solvents with a low boiling point and dielectric constants, such as acetone, the microcrystallization process occurs rapidly due to their fast evaporation and volatility. In this case, mechanical or any other driving forces that can break the droplets into smaller clusters should be suppressed, resulting in a similar number of small droplets, regardless of the concentration of the Schiff base compound in the organic solvent. In light of this consideration, the number of Schiff base molecules per unit droplet should be linearly correlated with the concentration.

The size of the formed microcrystals was measured using DLS after $10 \mathrm{~min}, 24 \mathrm{~h}$, and $48 \mathrm{~h}$ after the target compound was injected into the poor solvent medium. This resulted in almost no change in the size of the microcrystals, suggesting that the Schiff base microcrystals formed were stable.

We examined the effect of temperature on the crystal size by DLS analysis of two concentrations $(0.0005$ and $0.002 \mathrm{M})$ of the target compound dissolved in acetone. Figure $2 \mathrm{~b}$ shows a noticeable increase in the TASBnDTC crystal size as the reaction temperature increased. The average crystal sizes for both concentrations were drastically increased as the temperature surpassed the boiling point of the good solvent (acetone, b.p. $56^{\circ} \mathrm{C}$ ). This increasing trend is assumed to reflect the increase in the rate of evaporation of acetone as the temperature increases. When the good solvent evaporated at a faster rate, the fast contact of the dissolved target molecules with the water phase rapidly induced the formation of clusters, which led to the formation of microcrystals.

Generally, the presence of CTAB in the solution will result in the spontaneous formation of micelles within which the crystals grow (Dalavi et al., 2014). CTAB assembles itself as a layer micelle, where the alkylammonium group faces the surrounding water molecules and its hydrophobic region is arranged so that the chain is directed towards the center, away from the water molecules. As reported by Becker et al. (2010), as the temperature increases, the micelle structure becomes less stable. This causes the CTAB molecules to become more available to form the double-layered structure needed for the formation of the microcrystal. At $0.05 \%$, the CTAB solution is relatively highly concentrated and it is only soluble at temperatures above $25^{\circ} \mathrm{C}$. The $\mathrm{CTAB}$ gradually re-precipitates at temperatures lower than $25^{\circ} \mathrm{C}$; therefore, the effectiveness of the CTAB in reducing the crystal size is also decreased. As a result, the size of the TASBnDTC microcrystals was only slightly affected due to its bulky structure and lack of a reactive functional group (data not shown). 


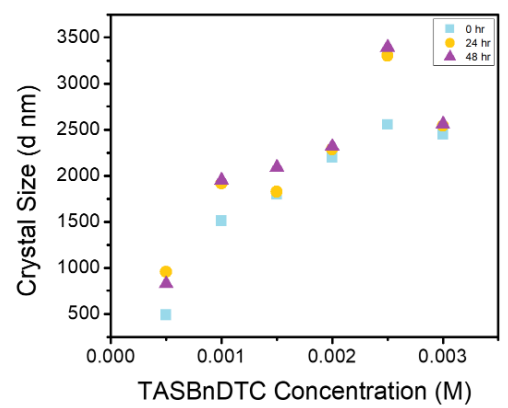

(a)

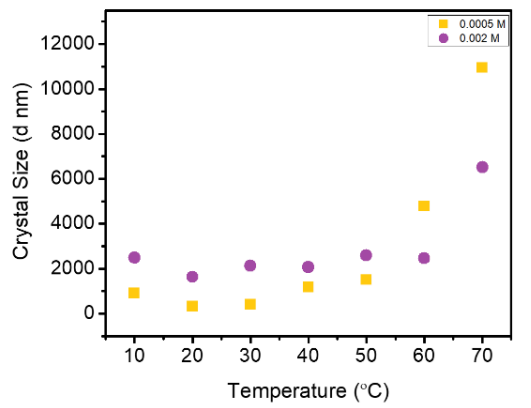

(b)

Figure 2 DLS analysis results showing: (a) TASBnDTC microcrystal size plotted against concentration of TASBnDTC dissolved in acetone in a span of 48 hours; and (b) The increasing trend of TASBnDTC microcrystal size as the temperature of the poor medium increases

\subsection{UV-Vis Spectra and X-Ray Powder Diffraction}

Figure 3a shows the UV-visible absorption spectra of different-sized surfactantdispersed TASBnDTC microcrystals $(1505,2194$, and $2447 \mathrm{~nm})$ fabricated from three different concentrations $(0.001 \mathrm{M}, 0.002 \mathrm{M}$, and $0.003 \mathrm{M})$ of the Schiff base in the acetonewater system. The spectrum of TASBnDTC dissolved in acetone is also shown for comparison. The obtained absorption spectra show slight changes at the tail end of the red, blue, and green spectra that are not seen in the molecular spectrum of TASBnDTC dissolved in acetone. These changes are due to Rayleigh scattering, confirming the formation of TASBnDTC microcrystals. The increase in absorbance as the concentration increases may reflect the increase in particle density. A hypsochromic shift at the microcrystals' excitonic peak is also produced. The electronic transition spectrum of molecular TASBnDTC was observed in the range of $370-450 \mathrm{~nm}$, and it was blue shifted to the $310-400 \mathrm{~nm}$ region when TASBnDTC was in the microcrystalline state. The hypsochromic shift, informally referred to as the blue shift, occurs as a result of intermolecular structural changes or disturbance of the electronic states due to environmental factors in the crystalline state (Burda et al., 2005).

The crystalline phase of the TASBnDTC microcrystals was investigated with XRD. The crystalline phase of the TASBnDTC microcrystals prepared by the reprecipitation method was similar, irrespective of the different TASBnDTC concentrations. The XRD peaks for the TASBnDTC microcrystals, as shown in Figure 3b, are observed at 7.1, 17.4, 20.8, 21.7, 23.3, 25.8, 27.8, 30.7 and $38.4^{\circ}$. Nonetheless, the peaks for TASBnDTC microcrystals differ slightly, suggesting different crystalline structures than in its bulk crystal.

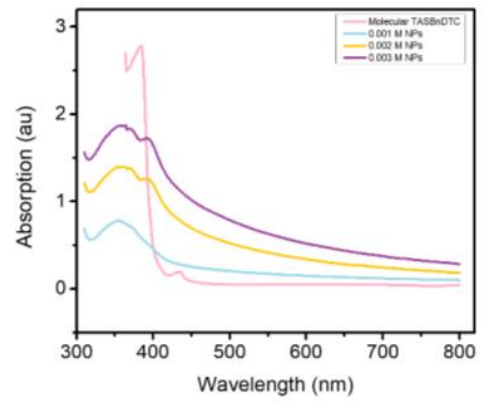

(a)

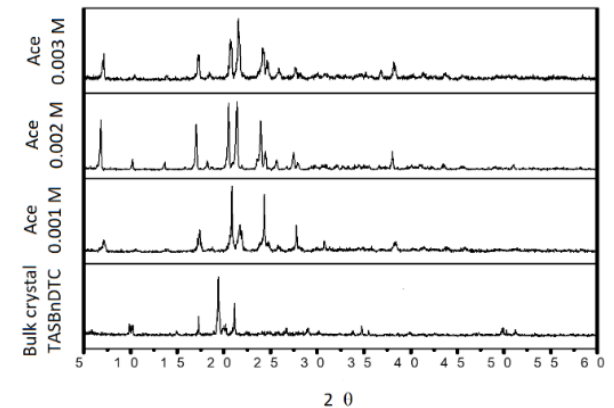

(b)

Figure 3 (a) UV-Visible absorption spectra of molecular TASBnDTC dissolved in acetone at concentration of $0.001 \mathrm{M}$ (pink) and its microcrystals at concentration of $0.001 \mathrm{M}$ (blue), $0.002 \mathrm{M}$ (yellow), and $0.003 \mathrm{M}$ (purple); and (b) XRD patterns of TASBnDTC bulk crystals and their microcrystals prepared in acetone at three different concentrations 


\subsection{Scanning Electron Microscopy (SEM)}

The surface morphology of TASBnDTC microcrystals prepared using the reprecipitation method at different concentrations is shown in their SEM images, as presented in Figure 4. TASBnDTC microcrystals have an irregular shape, almost resembling a distorted rectangular shape, with approximate sizes ranging from 800 to $2000 \mathrm{~nm}$. This size was similar to the sizes observed by DLS, regardless of the variation in the concentration of the target compound and the organic solvent used as the good solvent. This suggests that CTAB prevails as a surfactant and prevents the aggregation of the microcrystals into much larger sized microcrystals.

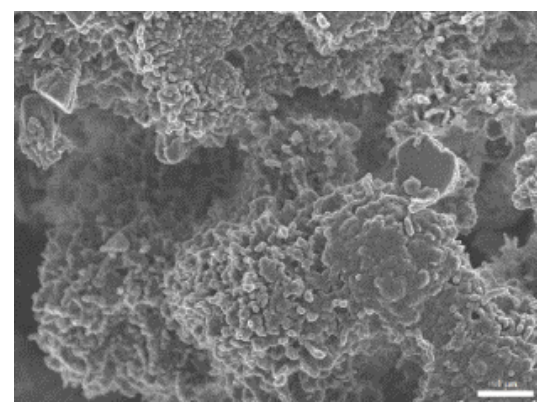

(a)

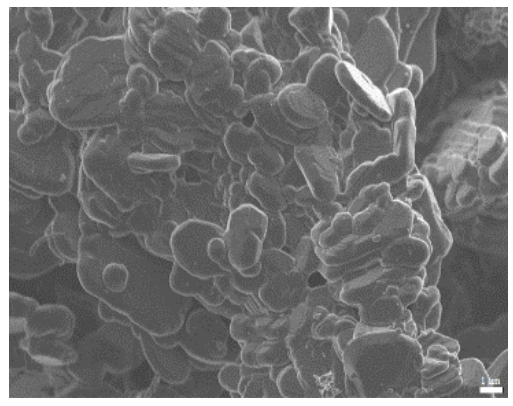

(b)

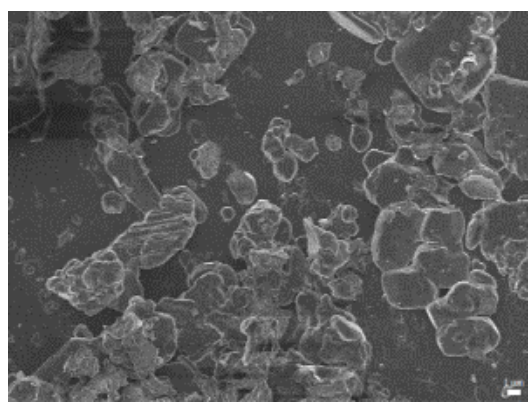

(c)

Figure 4 Representative SEM images of TASBnDTC microcrystals fabricated by the reprecipitation method in acetone-water system at TASBnDTC concentration (magnification) of: (a) $0.001 \mathrm{M}$ $(\times 15,000)$; (b) $0.002 \mathrm{M}(\times 8,000)$; and (c) $0.003 \mathrm{M}(\times 5,000)$. The scale bar shown denotes $1 \mu \mathrm{m}$ size

\subsection{Antibacterial Screening Activity}

The synthesized Schiff base and its microcrystals were screened in vitro for their antibacterial activity using four different bacteria; E. coli, B. subtilis, S. aureus, and $P$. aeruginosa. The concentration used for testing the antibacterial activity was $2 \mathrm{mg} / \mathrm{mL}$ for all the synthesized compounds and the positive control. The diameter of the zone of inhibition (a clear zone that indicates no bacterial growth) was measured and presented in Table 1. From the results obtained, the antibacterial activities of TASBnDTC microcrystals were enhanced at 9.25, 8.0, 9.6, and $10.75 \mathrm{~mm}$, for $S$. aureus, B. subtilis, E. coli, and $P$. aeruginosa, respectively, compared to the bulk molecular form, which showed no activity against any of the four bacterial strains. However, the inhibition was less than that achieved with the standard antibiotic streptomycin, which gave a zone of inhibition ranging from 18.0 to $23.0 \mathrm{~mm}$. This reflects the lower capability of the microcrystals as antibacterial agents; however, this preliminary study confirmed that when the Schiff base is in the form of microcrystals, its activity is enhanced due to the increase in surface area of the microcrystals (Yariv et al., 2015). In addition, organic microcrystals in the reprecipitation method exist as freestanding forms in a dispersion medium (Kasai et al., 2012); therefore, they have lower liposolubility and would reduce the ease of diffusion into the bacteria (Fasina et al., 2013).

Table 1 The antibacterial activities of the synthesized Schiff base and their microcrystals

\begin{tabular}{ccccc}
\hline \multirow{2}{*}{ Compounda } & \multicolumn{4}{c}{ Zone of inhibition, (mm) } \\
\cline { 2 - 5 } & S. aureus & B. subtilis & E. coli & P. aeruginosa \\
\hline TASBnDTC & NA & NA & NA & NA \\
TASBnDTC NPs 0.002 M & 9.25 & 8.0 & 9.6 & 10.75 \\
Streptomycin sulfate & 18 & 19 & 20 & 23 \\
\hline
\end{tabular}

aNA: No Activity 


\section{Conclusions}

This study provides some new insight into the microcrystallization of Schiff base compounds by the reprecipitation method using an organic solvent-water system. Adjusting the concentration of the target compound in the organic solution and the temperatures of the microcrystallization process was confirmed as a feasible and practical way to control the size and quality of the organic microcrystals. The rate and efficiency of dispersion of the organic solvent in water govern the microcrystal size. These insights into microcrystallization by the reprecipitation method would allow further development of organic microcrystal fabrication in the Rayleigh regime with fully controlled properties. Varying the concentration of TASBnDTC with acetone at $0.001,0.002$, and $0.003 \mathrm{M}$ yielded microcrystals of the compound with the same crystal structure. Interestingly, this preliminary study showed that these Schiff base microcrystals have slight antibacterial activities against four strains of bacteria (inhibition zone ranges of 8.0 to $10.75 \mathrm{~mm}$ ), whereas the bulk molecular form has no antibacterial activity at all. Therefore, the Schiff base microcrystals warrant further studies by varying the moiety of the Schiff base as well as exploring its minimum inhibition concentration. These studies are currently underway in our laboratories.

\section{Acknowledgements}

We are grateful to the Chemical Sciences [FIC Grant No. UBD/RSCH/1.4/FICBF(b)/2020/024], the Environmental and Life Sciences, Geosciences and Centre for Advanced Material and Energy Sciences, Universiti Brunei Darussalam for the necessary support in carrying out the research work.

\section{References}

Abyan, M., Bertorelle, F., Fery-Forgues, S., 2005. Use of Linear Polymers to Control the Preparation of Luminescent Organic Microcrystals. Langmuir, Volume 21(13), pp. 6030-6037

Ali, N.H.S.O., Hamid, M.H.S.A., Putra, N.A.A.M.A, Adol, H.A., Mirza, A.H., Usman, A., Siddiquee, T.A., Hoq, M.R., Karim, M.R., 2020. Efficient Eco-Friendly Syntheses of Dithiocarbazates and Thiosemicarbazones. Green Chemistry Letters and Reviews, Volume 13(2), pp. 129140

Baba, K., Kasai, H., Okada, S., Oikawa, H., Nakanishi, H., 2000. Novel Fabrication Process of Organic Microcrystals using Microwave-Irradiation. Japan Journal of Applied Physics, Volume 39(12A), pp. 1256-1258

Becker, R., Liedberg, B., Käll, P.O., 2010. CTAB Promoted Synthesis of Au NanorodsTemperature Effects and Stability Considerations. Journal of Colloid and Interface Science, Volume 343(1), pp. 25-30

Burda, C., Chen, X., Narayanan, R., El-Sayed, M.A., 2005. Chemistry and Properties of Nanocrystals of Different Shapes. Chemical Reviews, Volume 105(4), pp. 1025-1102

Chiang, H., Iimori, T., Onodera, T., Oikawa, H., Ohta, N., 2012. Gigantic Electric Dipole Moment of Organic Microcrystals Evaluated in Dispersion Liquid with Polarized Electroabsorption Spectra . The Journal of Physical Chemistry, Volume 116(14), pp. 8230-8235

Dalavi, D.K., Bhopate, D.P., Bagawan, A.S., Gore, A.H., Desai, N.K., Kamble, A.A., Mahajan, P.G., Kolekar, G.B., Patil, S.R., 2014. Fluorescence Quenching Studies of CTAB Stabilized Perylene Nanoparticles for the Determination of $\mathrm{Cr}(\mathrm{VI})$ from Environmental Samples: Spectroscopic Approach. Analytical Methods, Volume 6(17), pp. 6948-6955 
Fasina, T.M., Ejiah, F.N., Dueke-Eza, C.U., Idika, N., 2013. Substituent Effect on the Antimicrobial Activity of Schiff Bases Derived from 2-Aminophenol and 2Aminothiophenol. International Journal of Biological Chemistry, Volume 7, pp. 79-85

Indrasti, N.S., Ismayana, A., Maddu, A., Utomo, S.S., 2020. Synthesis of Nano-silica from Boiler Ash in the Sugar Cane Industry using the Precipitation Method. International Journal of Technology, Volume 11(2), pp. 422-435.

Jahangirian, H., Haron, M.J., Ismail, M.H.S., Rafiee-Moghaddam, R., Afsah-Hejri, L., Abdollahi, Y., Rezayi, M., Vafaei, N., 2013. Well Diffusion Method for Evaluation of Antibacterial Activity of Copper Phenyl Hydroxamate Synthesized from Canola and Palm Kernel Oils. Digest Journal of Nanomaterials and Biostructures, Volume 8(3), pp. 1263-1270

Jortner, J., Rao, C.N.R., 2002. Nanostructured Advanced Materials. Perspectives and Directions. Pure and Applied Chemistry, Volume 74(9), pp. 1491-1506

Kasai, H., Nakanishi, H., Oikawa, H., 2012. Application 42 - Fabrication Technique of Organic Nanocrystals and Their Optical Properties and Materialization. In: Nanoparticle Technology Handbook, Hosokawa M., Nogi, K., Naito, M., Yokoyama, T. (eds.), Elsevier, Amsterdam, pp. 596-601

Kasai, H., Nalwa, H.S., Oikawa, H., Okada, S., Matsuda, H., Minami, N., Kakuta, A., Ono, K., Mukoh, A., Nakanishi, H., 1992. A Novel Preparation Method of Organic Microcrystals. Japan Journal of Applied Physics, Volume 31(8A), pp. 1132-1134

Komai, Y., Kasai, H., Hirakoso, H., Hakuta, Y., Katagi, H., Okada, S., Oikawa, H., Adschiri, T., Inomata, H., Arai, K., Nakanishi, H., 1999. Preparation of Organic Microcrystals using Supercritical Fluid Crystallization Method Preparation of Organic. Japan Journal of Applied Physics, Volume 38(1A), pp. 81-83

Kumar, R., Lal, S., 2014. Synthesis of Organic Nanoparticles and their Applications in Drug Delivery and Food Nanotechnology: A Review. Journal of Nanomaterials \& Molecular Nanotechnology, Volume 3(4), pp 1-11

Kumar, S., Mittal, S.K., Kaur, N., 2019. Enhanced Performance of Organic Nanoparticles of a Schiff Base for Voltammetric Sensor of $\mathrm{Cu}(\mathrm{II})$ Ions in Aqueous Samples. Analytical Methods, Volume 11(3), pp. 359-366

Li, W., Baren, J.V., Berges, A., Bekyarova, E., Lui, C.H., Bardeen, C.J., 2020. Shaping Organic Microcrystals using Focused Ion Beam Milling. Crystal Growth and Design, Volume 20(3), pp. 1583-1589

Li, Z.Z., Liang, F., Zhuo, M.P., Shi, Y.L., Wang, X.D., 2017. White-Emissive Self-Assembled Organic Microcrystals. Small, Volume 13(19), pp. 1-6

Magdassi, S., Moshe, M.B., 2003. Patterning of Organic Nanoparticles by Ink-Jet Printing of Microemulsions. Langmuir, Volume 19(3), pp. 939-942

Mahajan, P.G., Kolekar, G.B., Patil, S.R., 2017. Recognition of D-Penicillamine using Schiff Base Centered Fluorescent Organic Nanoparticles and Application to Medicine Analysis. Journal of Fluorescence, Volume 27(3), pp. 829-839

Malik, M.A., Lone, S.A., Wani, M.Y., Talukdar, M.I.A., Dar, O.A., Ahmad, A., Hashmi, A.A., 2020. S-Benzyldithiocarbazate Imine Coordinated Metal Complexes kill Candida albicans by causing Cellular Apoptosis and Necrosis. Bioorganic Chemistry, Volume 98, pp. 1-11

Miyashita, Y., Baba, K., Kasai, H., Nakanishi, H., Miyashhita, T., 2008. A New Production Process of Organic Pigment Nanocrystals. Molecular Crystals and Liguid Crystals, Volume 492(1), pp. 268/[632]-274/[638]

Mondal, S., Mandal, S.M., Mondal, T.K., Sinha, C., 2017. Spectroscopic Characterization, Antimicrobial Activity, DFT Computation and Docking Studies of Sulfonamide Schiff Bases. Journal of Molecular Structure, Volume 1127, pp. 557-567 
Oikawa, H., Fujita, S., Kasai, H., Okada, S., 2000. Electric Field-Induced Orientation of Organic Microcrystals with Large Dipole Moment in Dispersion Liquid. Colloid and Surfaces A: Physicochemical and Engineering Aspects, Volume 169(1-3), pp. 251-258

Patil, K.S., Mahajan, P.G., Patil, S.R., 2017. Fluorimetric Detection of $\mathrm{Sn}^{2+}$ Ion in Aqueous Medium using Salicylaldehyde Based Nanoparticles and Application to Natural Samples Analysis. Spectrochimica Acta-Part A: Molecular and Biomolecular Spectroscopy, Volume 170, pp. 131-137

Poerwadi, B., Kartikowati, C.W., Oktavian, R., Novaresa, O., 2020. Manufacture of a Hydrophobic Silica Nanoparticle Composite Membrane for Oil-Water Emulsion Separation. International Journal of Technology, Volume 11(4), pp. 364-373

Rahman, S.N.S.A., 2018. Fabrications of Polycyclic Aromatic Hydrocarbon and Schiff Bases Nanocrystals by Reprecipitation Method. Master's Thesis, MSc Program, Universiti Brunei Darussalam, Brunei Darussalam

Raj, S.S.S., Yamin, B.M., Yussof, Y.A., Tarafder, M.T.H., Fun, H.-K., Grouse, K.A., 2000. TransCis S-Benzyl Dithiocarbazate. Acta Crystallographica Section E Crystal Structure Communications, Volume 56(10), pp. 1236-1237

Strokova, V.V, Baskakov, P.S., Ayzenshtadt, A.M., Nelyubova, V.V., 2020. Creation of Biocidal Coatings using the Stabilization of Silver Nanoparticles in Aqueous Acrylic Dispersions. International Journal of Technology, Volume 11(1), pp. 5-14

Tripathy, S.K., Katagi, H., Kasai, H., Balasubramanian, S., Oshikiri, H., Kumar, J., Oikawa, Hidetoshi, O., Okada, S., Nakanishi, H., 1998. Self Assembly of Organic Microcrystals 1: Electrostatic Attachment of Polydiacetylene Microcrystals on a Polyelectrolyte Surface. Japan Journal of Applied Physics, Volume 37(2-3B), pp. 343-345

Yariv, I., Lipovsky, A., Gedanken, A., Lubart, R., Fixler, D., 2015. Enhanced Pharmacological Activity of Vitamin B12 and Penicillin as Nanoparticles. International Journal of Nanomedicine, Volume 10(1), pp. 3593-3601 